\title{
Clinical Study \\ Parameters Associated with Significant Liver Histological Changes in Patients with Chronic Hepatitis B
}

\author{
Li Xiao, Jianchun Xian, Yang Li, Aiwen Geng, Xiuzhen Yang, Libin Han, and Hongtao Xu \\ Department of Liver Diseases, Taizhou People’s Hospital, Jiangsu 225300, China \\ Correspondence should be addressed to Hongtao Xu; 15850850979@163.com
}

Received 20 October 2013; Accepted 12 November 2013; Published 28 January 2014

Academic Editors: W. Vogel and J. Yu

Copyright ( 2014 Li Xiao et al. This is an open access article distributed under the Creative Commons Attribution License, which permits unrestricted use, distribution, and reproduction in any medium, provided the original work is properly cited.

\begin{abstract}
This study aimed to evaluate factors associated with significant liver histological changes. Liver biopsies from $157 \mathrm{CHB}$ patients were retrospectively analyzed. Only ALB was significantly correlated with advanced liver necroinflammatory $(P=0.001)$. Age, ALB, GLOB, AST, PLT, and PT were independent predictors of significant fibrosis $(P=0.002, P<0.001, P=0.001, P=0.048$, $P<0.001$, and $P=0.001$, resp.). AST, WBC, and HBV DNA were significantly correlated with advanced fibrosis in normal ALT patients $(P<0.001, P=0.041$, and $P=0.012$, resp. $)$ and age, ALB, GLOB, PLT, and PT in patients with abnormal ALT $(P=0.003$, $P<0.001, P=0.004, P<0.001$, and $P=0.002$, resp.). Age, AST, GGT, PLT, and PT were significantly associated with advanced fibrosis in HBeAg+ patients $(P=0.01, P=0.016, P=0.027, P=0.016$, and $P=0.009$, resp. $)$ and ALB, GLOB, WBC, PLT, and PT in HBeAg- patients $(P<0.001, P=0.004, P=0.005, P<0.001$, and $P=0.035$, resp.). PLT was an excellent predictor for cirrhosis $(P<0.001$ and AUROC $=0.805)$. ALT was not predictive of advanced fibrosis for patients with $\mathrm{HBeAg}+\mathrm{or} \mathrm{HBeAg}-(P=0.273$ and $P=0.599$, resp.). PLT was an excellent predictor for cirrhosis in CHB patients. Liver histopathology can be recommended for chronic HBV carriers of older age, with normal ALT, lower PLT, and lower ALB.
\end{abstract}

\section{Introduction}

Globally, there are approximately 350-400 million people infected with chronic hepatitis B virus (CHB) [1] and in China, a high endemic area, there an estimated 93 million people infected [2]. Without treatment, 15 to $40 \%$ of people with chronic HBV develop cirrhosis with a risk of developing hepatocellular carcinoma (HCC) $[3,4]$. The clinical course of chronic HBV infection ranges from an inactive carrier state to cirrhosis, hepatic decompensation, and HCC [5-7]. Fibrosis often evolves insidiously, especially in inactive HBV carriers [8]. Active inflammation appears to be the driving force for development of fibrosis [9].

Liver biopsy remains the investigation of choice for assessment of inflammation and fibrosis. Liver biopsy is recommended for certain patients with chronic HBV infection especially older patients, those with persistent HBV DNA levels above 2,000 IU/mL and ALT 1-2 $\times$ the upper limit of normal (ULN), according to the Asian Pacific Association for the Study of the Liver (APASL), the European Association for the Study of the Liver (EASL), and the American
Association for the Study of Liver Diseases (AASLD) [1012]. Antiviral treatment is recommended when liver histology reveals moderate-to-severe active necroinflammation and/or significant fibrosis by METAVIR scoring [10-12]. Although liver biopsy is often essential in the management of patients with liver disease, physicians and patients are concerned about the invasive nature of the procedure and potential complications [13-15]. Sampling error and intraobserver variations are also not infrequent $[16,17]$. Therefore, the development of noninvasive markers for significant liver disease is an attractive supplementary tool. Some noninvasive methods are used to assess liver fibrosis, such as transient elastography (TE) (FibroScan), FibroTest-ActiTest (Biopredictive, Labcorp) [14], and Fibrospect II (Prometheus) [9]. However, noninvasive methods are expensive and/or require equipment that is not widely available. These predictive markers of advanced liver pathology are useful screening tools that complement liver biopsy or help identify highrisk patients who are unwilling to undergo a liver biopsy. Several recent studies reported a relationship between liver function tests, HBV DNA levels, clinical characteristics, and 
other parameters with liver fibrosis [18-22]. However, these studies were limited by either a small number of parameters or only contained specific groups of patients, for example, with $\mathrm{HBeAg}+$ and $\mathrm{HBeAg}-$ or normal and abnormal ALT. To fully analyze the relationship between these parameters and liver histology in different patient groups, we retrospectively analyzed HBeAg status, ALT levels, age, and HBV DNA from 157 patients with chronic HBV infection who underwent liver biopsy from Oct 2009 to Dec 2012 at Taizhou People's Hospital, Jiangsu, China.

\section{Methods}

2.1. Patients. Entry criteria to the study included treatmentnaïve patients with chronic HBV infection who had a liver biopsy between Oct 2009 and Dec 2012 at the Taizhou People's Hospital, Jiangsu, China. Patients were recruited if they were positive for hepatitis B surface antigen ( $\mathrm{HBsAg}$ ) for at least 6 months, with an HBV DNA level of more than 500 copies $/ \mathrm{mL}$ and a prothrombin time of less than $18 \mathrm{sec}$. We excluded patients (1) with viral coinfections, including HCV, HDV, and HIV infection, (2) with decompensated liver disease, (3) with metabolic or autoimmune liver disease, and (4) with a history of hepatotoxic drug ingestion and a high alcohol intake (20 grams per day for female, 30 grams per day for male). Written consent was obtained prior to liver biopsy.

Fasting serum samples were tested one day prior to liver biopsy for laboratory parameters by standard methods. Fifteen parameters including clinical, biochemical, and hematological variables were analyzed: age, gender, $\mathrm{HBeAg}$ status, HBV DNA level, PT (prothrombin time), ALB (albumin), GLOB (globulin), TB (total bilirubin), TP (total protein), ALT (alanine aminotransferase), AST (aspartate aminotransferase), ALP (alkaline phosphatase), GGT (glutamyl transferase), PLT (platelet count), and WBC (white blood cell). Hepatitis B serology including $\mathrm{HBsAg} / \mathrm{Ab}, \mathrm{HBeAg} / \mathrm{Ab}$, and $\mathrm{HBCAb}$ was detected by enzyme-linked immunosorbent assay (Fosun Pharmaceutical Co., Shanghai, China). HBV DNA level was determined with a lower limit of quantification of 500 copies $/ \mathrm{mL}$ (about $100 \mathrm{IU} / \mathrm{mL}$ ) by real-time polymerase chain reaction (PCR) (Fosun Pharmaceutical Co., Shanghai, China).

The main equipments used in this study included $\mathrm{ABI}$ Real-time PCR analyzer 7500 and BioTek Synergy2 MultiMode Microplate Reader, USA, HITACHI Biochemical analyzer 7600, and automatic blood analyzer Sysmex XT-2000i, Japan.

2.2. Liver Biopsy. Ultrasonographic-guided liver biopsies were performed using $16 \mathrm{G}$ disposable needles (TSK corporation, Japan). Histological grading of necroinflammation and staging of liver fibrosis were considered reliable when the liver specimen length was $\geq 15 \mathrm{~mm}$ or the portal tract number $\geq 10$ [23]. The histological diagnosis was established using hematoxylin and eosin (H\&E) staining and Masson's trichrome stains of formalin fixed paraffin-embedded liver tissue. Two highly experienced liver pathologists reviewed all the liver specimens blinded to the patients' laboratory

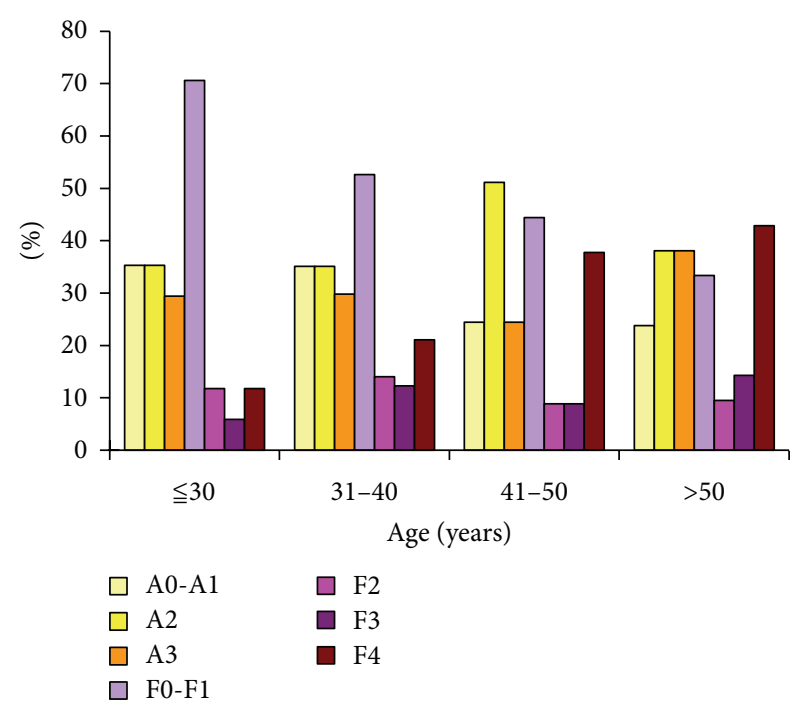

FIGURE 1: Distribution of liver necroinflammation and liver fibrosis (based on Metair Score) among different age groups in 157 patients with chronic HBV infection.

data. Histopathological findings were assessed and scored according to the METAVIR scoring system: $\mathrm{A} 0=$ no activity, $\mathrm{A} 1=$ mild activity, $\mathrm{A} 2=$ moderate activity, and $\mathrm{A} 3=$ severe activity; F0 = no fibrosis, F1 = portal fibrosis without septa, F2 $=$ portal fibrosis with rare septa, $\mathrm{F} 3=$ numerous septa without cirrhosis, and F4 = cirrhosis [24].

2.3. Statistical Analysis. The baseline data of patients were presented as the median. Statistical analysis was carried out with SPSS 13.5 software for windows (SPSS Inc., Chicago, IL, USA). Chi-square test was used for categorical variables, the Student $t$-test for numerical variables, and logistic regression analysis to further test whether the identified variables associated with advanced histological abnormalities were independent risk factors. Spearman's rank correlation was used to assess correlation between variables, liver necroinflammation grades, and liver fibrosis stages. All $P$ values were two sided and considered as statistically significant if $<0.05$.

\section{Results}

3.1. Patient Data. A total of 157 patients with all 15 clinical parameters available were enrolled into the study. There were 48 patients with inflammatory active grade A0-A1 and 109 at $\mathrm{A} 2-\mathrm{A} 3$ and 81 patients at liver fibrosis stage $\mathrm{F} 0-\mathrm{F} 1$ and 76 at F2-F4. The characteristics of all 157 patients are shown in Table 1.

A total of 34 patients were aged $\leqq 30,57$ aged $30-40$, 45 aged $40-50$, and 21 aged $>50$. There was no significant difference in necroinflammation grades among different age groups $(P=0.120)$; however, increasing age was independently associated with significant fibrosis $(P=0.034)$ (Figure 1).

The presence of $\mathrm{HBeAg}$ and hepatitis B viral load decreased with age: $28(82 \%)$ and $28(82 \%)$ had HBV DNA 
TABLE 1: Characteristics of 157 patients included in the study.

\begin{tabular}{|c|c|c|c|c|c|}
\hline Variables & Total & A0-A1 & A2-A3 & F0-F1 & $\mathrm{F} 2-\mathrm{F} 4$ \\
\hline Number of patients & 157 & 48 & 109 & 81 & 76 \\
\hline Age (years) & $38(14-68)$ & $36(14-58)$ & $39(18-68)$ & $36(14-64)$ & $41(25-68)$ \\
\hline Gender (male) & $136(87 \%)$ & $40(83 \%)$ & $96(91 \%)$ & $68(84 \%)$ & $68(89 \%)$ \\
\hline $\mathrm{HBeAg}+$ & $68(43 \%)$ & $21(44 \%)$ & $47(43 \%)$ & $41(51 \%)$ & $27(36 \%)$ \\
\hline HBeAg- & $89(57 \%)$ & $27(56 \%)$ & $62(57 \%)$ & $40(49 \%)$ & $49(64 \%)$ \\
\hline HBV DNA (log copies/mL) & $6(2-9)$ & $5.5(3-9)$ & $6(2-9)$ & $6(2-9)$ & $5.5(2-8)$ \\
\hline $\mathrm{PT}(\mathrm{sec})$ & $13(8.2-17.8)$ & $13(8.2-16)$ & $13.1(9.2-17.8)$ & $12.8(8.2-16.7)$ & $13.7(9.5-17.8)$ \\
\hline $\operatorname{ALB}(g / L)$ & $42.5(30.1-53.9)$ & $43.3(33.5-53.7)$ & $42(30.1-53.9)$ & $43.3(33.3-53.9)$ & $41.15(30.1-48.9)$ \\
\hline GLOB $(g / L)$ & $31.2(21.9-48.2)$ & $30.5(23.3-48.1)$ & $31.9(21.9-48.2)$ & $30.2(21.9-45.9)$ & $33.1(23.9-48.2)$ \\
\hline TB (umol/L) & $16.2(5.9-218)$ & $14.5(6.1-84.7)$ & $16.3(5.9-218)$ & $14.3(5.9-218)$ & $18.1(6.1-170.5)$ \\
\hline $\mathrm{TP}(\mathrm{g} / \mathrm{L})$ & $74.1(59.5-92.5)$ & $75.3(64.4-92.5)$ & $74(59.5-87.1)$ & $74(60.6-92.5)$ & $74.6(59.5-89.3)$ \\
\hline $\operatorname{ALT}(\mathrm{IU} / \mathrm{L})$ & $69(13-1387)$ & $64(18-1387)$ & $72(13-1215)$ & $62(13-1387)$ & $71(17-999)$ \\
\hline AST (IU/L) & $46(14-723)$ & $44.5(16-658)$ & $47(14-723)$ & $40(14-571)$ & $58(17-723)$ \\
\hline ALP (IU/L) & $79(35-323)$ & $73(35-323)$ & $80(41-258)$ & $74(35-323)$ & $88(42-258)$ \\
\hline GGT (IU/L) & $43(7-476)$ & $38(7-467)$ & $46(11-402)$ & $30(7-476)$ & $62(15-402)$ \\
\hline $\operatorname{PLT}\left(\times 10^{9} / \mathrm{L}\right)$ & $115(19-314)$ & $122.5(43-257)$ & $114(19-314)$ & $132(28-257)$ & $97(19-314)$ \\
\hline WBC $\left(\times 10^{9} / \mathrm{L}\right)$ & $4.69(1.96-7.77)$ & $4.68(2.73-7.64)$ & $4.7(1.96-7.77)$ & $5.11(2.4-7.77)$ & $4.32(1.96-7.64)$ \\
\hline
\end{tabular}

Continuous variables are expressed as the median (range).

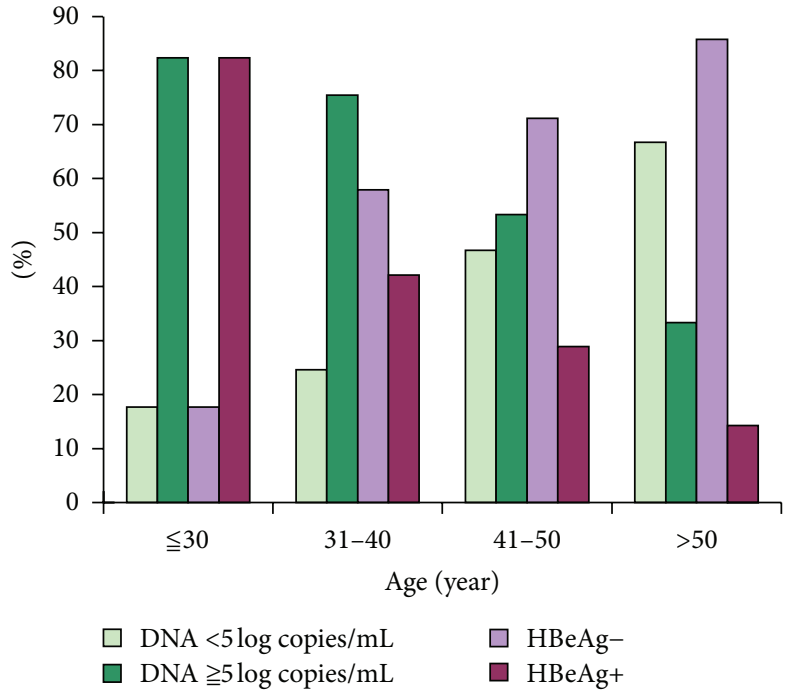

FIgure 2: Characteristic of HBV DNA and HBeAg status among different age groups in 157 patients with chronic HBV infection.

$\geqq 5 \log$ copies/mL and $\mathrm{HBeAg}+$, respectively, among the 34 patients aged $\leqq 30$. Seven $(33 \%)$ and $3(14 \%)$ had HBV DNA $\geqq 5 \log$ copies/mL and $\mathrm{HBeAg}+$, respectively, among the 21 patients aged $>50$ (Figure 2). The presence of HBeAg was associated with higher HBV DNA $(P<0.001)$.

A total of 31 patients (19.7\%) had ALT levels within the normal range suggested by Prati et al. [25] (i.e., $30 \mathrm{U} / \mathrm{L}$ for men and $19 \mathrm{U} / \mathrm{L}$ for women) and 126 patients exceeded the Prati criteria. Nine of 31 (29\%) patients with normal ALT had cirrhosis and 33 of 126 (26\%) patients with abnormal ALT had cirrhosis. There was no significant difference in liver necroinflammation grades or fibrosis stages between

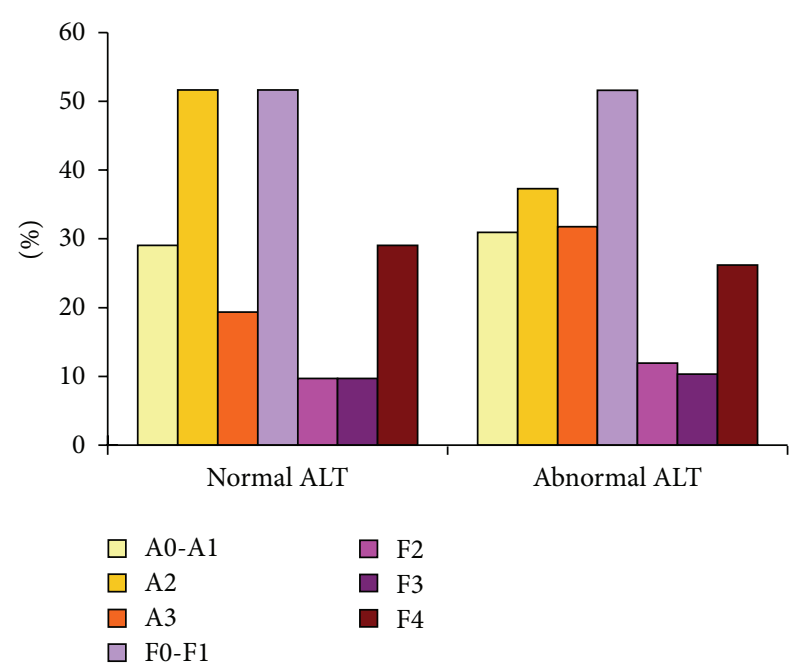

FIGURE 3: The distribution of liver necroinflammation and fibrosis in patients with normal and abnormal ALT.

patients with normal and abnormal ALT $(P=0.835$ and $P=$ 0.998, resp.) (Figure 3). Spearman's rank correlation showed that AST, WBC, and DNA were significantly correlated with advanced fibrosis in patients with normal ALT $(P<0.001$, $P=0.041$, and $P=0.012$, resp.), while age, ALB, GLOB, PLT, and PT were significantly correlated with advanced fibrosis in patients with abnormal ALT $(P=0.003, P<0.001$, $P=0.004, P<0.001$, and $P=0.002$, resp.).

Advanced necroinflammation was found in $39.8 \%$ of $\mathrm{HBeAg}+$ patients and $55.1 \%$ of $\mathrm{HBeAg}-$ patients. Advanced fibrosis was found in $62.2 \%$ of $\mathrm{HBeAg}+$ patients and in $69.7 \%$ of $\mathrm{HBeAg}-$ patients. There was no significant difference between $\mathrm{HBeAg}+$ and $\mathrm{HBeAg}-$ groups in necroinflammation 
TABLE 2: Clinical parameters predictive of advanced liver necroinflammation and fibrosis.

\begin{tabular}{|c|c|c|c|c|c|c|c|c|c|}
\hline & & \multicolumn{2}{|c|}{$t$ test } & \multicolumn{2}{|c|}{ Spearman's correlation } & \multicolumn{4}{|c|}{ Logistic regression } \\
\hline & & \multirow{2}{*}{$t$ value } & \multirow{2}{*}{$P$ value } & \multirow{2}{*}{ Correlation coefficient } & \multirow{2}{*}{$P$ value } & \multirow{2}{*}{ OR value } & \multirow{2}{*}{$P$ value } & \multicolumn{2}{|c|}{$95 \% \mathrm{CI}$ for OR } \\
\hline & & & & & & & & Lower & Upper \\
\hline \multirow[t]{4}{*}{$\mathrm{A} 2-\mathrm{A} 3$} & ALB & 3.238 & 0.001 & -0.236 & 0.003 & 0.888 & 0.002 & 0.823 & 0.958 \\
\hline & Age & -3.075 & 0.002 & 0.245 & 0.002 & 2.113 & 0.002 & 1.330 & 3.356 \\
\hline & ALB & 4.099 & $<0.001$ & -0.291 & $<0.001$ & 0.119 & 0.037 & 0.016 & 0.879 \\
\hline & GLOB & -3.282 & 0.001 & 0.249 & 0.002 & 0.136 & 0.049 & 0.019 & 0.988 \\
\hline \multirow[t]{3}{*}{$\mathrm{F} 2-\mathrm{F} 4$} & AST & -1.997 & 0.048 & 0.235 & 0.003 & 1.031 & 0.011 & 1.007 & 1.055 \\
\hline & PLT & 4.548 & $<0.001$ & -0.383 & $<0.001$ & 0.991 & 0.037 & 0.982 & 0.999 \\
\hline & PT & -3.355 & 0.001 & 0.256 & 0.001 & 1.641 & $<0.001$ & 1.252 & 2.151 \\
\hline
\end{tabular}

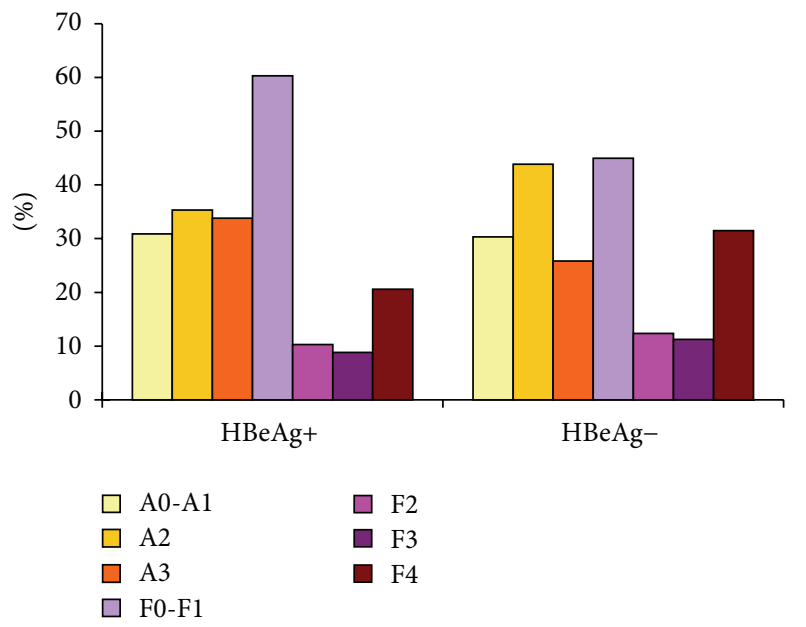

FIGURE 4: Distribution of liver necroinflammation and liver fibrosis (based on Metair Score) among HBeAg+ and HBeAg- groups.

grades and fibrosis stages $(P=0.057$ and $P=0.941$, resp.) (Figure 4). Age, AST, GGT, PLT, and PT were significantly correlated with advanced fibrosis in $\mathrm{HBeAg}+$ patients $(P=$ 0.01, $P=0.016, P=0.027, P=0.016$, and $P=0.009$, resp.). ALB, GLOB, WBC, PLT, and PT were significantly correlated with advanced fibrosis in $\mathrm{HBeAg}-$ patients $(P<0.001, P=$ $0.004, P=0.005, P<0.001$, and $P=0.035$, resp.). There was no significant difference in fibrosis stages in patients with normal and abnormal ALT for both HBeAg+ and HBeAggroups $(P=0.273$ and $P=0.599$, resp. $)$.

3.2. Identification of Variables Predicting Advanced Liver Necroinflammation and Fibrosis. Two levels of liver fibrosis (F0-F1 and F2-F4) and liver necroinflammation (A0-A1 and A2-A3) were analyzed in 157 patients with chronic $\mathrm{HBV}$ infection. Of the 15 variables, only ALB was significantly correlated with advanced liver necroinflammation $(\mathrm{OR}=0.888 \mathrm{P}=$ 0.002). Spearman's rank correlation analysis showed that 10 variables (age, TB, ALB, GLOB, AST, ALP, GGT, WBC, PLT, and PT) were correlated significantly with advanced liver fibrosis (correlation coefficients were 0.245, 0.213, -0.291, $0.249,0.235,0.223,0.288,-0.258,-0.383$, and 0.256 , resp.; $P$ values were $0.002,0.007,<0.001,0.002,0.003,0.005,<0.001$, $0.001,<0.001$, and 0.001 resp.). Univariate analysis and logistic
TABLE 3: The area under ROC curve (AUC) of the identified variables for cirrhosis in all patients.

\begin{tabular}{lccc}
\hline & \multirow{2}{*}{ AUC for cirrhosis } & \multicolumn{2}{c}{$95 \%$ confidence interval } \\
& & Lower & Upper \\
\hline Age & 0.728 & 0.610 & 0.846 \\
ALB & 0.721 & 0.600 & 0.841 \\
GLOB & 0.760 & 0.645 & 0.875 \\
AST & 0.681 & 0.554 & 0.808 \\
PLT & 0.805 & 0.702 & 0.908 \\
PT & 0.708 & 0.581 & 0.834 \\
\hline
\end{tabular}

regression analysis revealed that only age, ALB, GLOB, AST, PLT, and PT were independent predictive factors and were significantly different in mild and moderate/severe fibrosis (Table 2). A lower ALB was independently associated with significant necroinflammation. Older age, higher AST, longer PT, lower PLT, and lower ALB were independently associated with significant fibrosis.

The area under ROC curve (AUC) of ALB for significant necroinflammation in all patients was 0.614 . The $95 \%$ confidence interval was 0.501 to 0.727 . PLT was a good predictor for cirrhosis and its AUROC was 0.805. The AUC of the predictors for cirrhosis was shown in Table 3.

\section{Discussion}

The development of noninvasive markers for liver fibrosis is an attractive option because of the associated risks of liver biopsy. Previous studies have shown inconsistent results and the optimal predictor for significant liver fibrosis is not known. This study investigated the association of various routinely available clinical parameters with liver histology.

Current guidelines for antiviral treatment of chronic HBV infection recommend therapy when ALT levels are more than twice the upper limit of normal; however, previous studies have not demonstrated a correlation between ALT levels and liver fibrosis [26-29]. We investigated the association between ALT, liver fibrosis, and necroinflammation and consistent with previous studies, found no significant correlation between ALT, liver fibrosis, necroinflammation, or with $\mathrm{HBe}$ status. We found that $29 \%$ of patients with normal ALT levels, using the reference ranges suggested by Prati et al. [25], had significant fibrosis. Therefore, using the ALT threshold of 
more than $2 \times U L N$, current guidelines may deny patients treatment, who have significant fibrosis or cirrhosis.

We found no significant association between $\mathrm{HBeAg}$ status and liver fibrosis. HBeAg status was, however, associated with higher HBV DNA levels and the presence of $\mathrm{HBeAg}$ and hepatitis B viral load decreased with age. The correlation between HBV DNA levels and liver fibrosis is controversial. Croagh et al. reported that HBV DNA was an independent predictor for significant fibrosis in $\mathrm{HBeAg}-$ but not $\mathrm{HBeAg}+$ patients [20]. Seto et al. reported that HBV DNA levels had no correlation with liver histology, but the majority of the study population contained $\mathrm{HBeAg}+$ patients [30]. Our study did not show any association between HBV DNA and liver histology in patients testing HBeAg+ nor HBeAg-. However, HBV DNA was significantly correlated with advanced fibrosis in patients with normal ALT. This may be explained by different ethnic groups, patient sample differences, HBV genotypes, and transmission route of HBV infection. Chinese mainland patients acquire the infection perinatally with liver injury starting early in life and the $\mathrm{HBV}$ genotypes are mainly B and C [31].

Most previous studies have focused on predictors for liver fibrosis and the main driving force for the development of fibrosis appears to be active inflammation. In the present study, we examined routine parameters for predicting liver necroinflammatory disease. Our study showed that only ALB among the 15 variables was negatively correlated with advanced liver necroinflammatory disease. Age, ALB, AST, PLT, and PT were independently associated with significant fibrosis in different chronic HBV groups, that is, $\mathrm{HBeAg}-$ and HBeAg+ patients [21, 26, 32-35]. Age is an important predictor and reflects progression of fibrosis in a time-dependent manner. Our study found age, ALB, GLOB, AST, PLT, and PT were associated with significant fibrosis in all patients with chronic HBV infection. AST had the lowest association with fibrosis, whereas PLT was an excellent predictor for significant fibrosis. A low platelet count is associated with advanced liver fibrosis through the altered production of thrombopoietin and is independent of demographic and biochemical characteristics, hepatic necroinflammatory activity, portal hypertension, and splenomegaly [36].

There are limitations of this study. HBV genotyping was not performed, which may have affected the results as previous small-scale studies found genotype $\mathrm{C}$ was associated with significant histological abnormalities [33, 37].

In conclusion, ALT is a poor marker when considering antiviral therapy because of its poor correlation with significant liver injury in patients with chronic HBV infection. Lower levels of PLT were independently associated with significant fibrosis. If a liver biopsy is considered to assess disease activity and fibrosis, it can be recommended for patients with chronic HBV infection, particularly for an older age group and patients with normal ALT and lower PLT and ALB.

\section{Conflict of Interests}

The authors have declared that no competing interests exist.

\section{Acknowledgment}

The authors have been supported by Science and Research Project Fund of Jiangsu Provincial Health Department No. H201059.

\section{References}

[1] J. L. Dienstag, "Drug therapy: hepatitis B virus infection," The New England Journal of Medicine, vol. 359, no. 14, pp. 1486-1500, 2008.

[2] X. Li, L. Wang, Y. Zhong et al., "Hepatitis B virus (HBV) subgenotypes $\mathrm{C} 2$ and B2 differ in lamivudine- and adefovirresistance-associated mutational patterns in HBV-infected Chinese patients," Journal of Clinical Microbiology, vol. 48, no. 12, pp. 4363-4369, 2010.

[3] B. J. McMahon, "Natural history of chronic hepatitis B-clinical implications," Medscape General Medicine, vol. 10, no. 4, article 91, 2008.

[4] M.-W. Yu, F.-C. Hsu, I.-S. Sheen et al., "Prospective study of hepatocellular carcinoma and liver cirrhosis in asymptomatic chronic hepatitis B virus carriers," American Journal of Epidemiology, vol. 145, no. 11, pp. 1039-1047, 1997.

[5] M. Manno, C. Cammà, F. Schepis et al., "Natural history of chronic HBV carriers in Northern Italy: morbidity and mortality after 30 years," Gastroenterology, vol. 127, no. 3, pp. 756-763, 2004.

[6] M. Martinot-Peignoux, N. Boyer, M. Colombat et al., "Serum hepatitis B virus DNA levels and liver histology in inactive HBsAg carriers," Journal of Hepatology, vol. 36, no. 4, pp. 543$546,2002$.

[7] M.-F. Yuen, "Revisiting the natural history of chronic hepatitis B: impact of new concepts on clinical management," Journal of Gastroenterology and Hepatology, vol. 22, no. 7, pp. 973-976, 2007.

[8] C.-M. Chu and Y.-F. Liaw, "Incidence and risk factors of progression to cirrhosis in inactive carriers of hepatitis B virus," American Journal of Gastroenterology, vol. 104, no. 7, pp. 16931699, 2009.

[9] H. Mani and D. E. Kleiner, "Liver biopsy findings in chronic hepatitis B," Hepatology, vol. 49, supplement 5, pp. S61-S71, 2009.

[10] Y.-F. Liaw, N. Leung, J.-H. Kao et al., "Asian-Pacific consensus statement on the management of chronic hepatitis B: a 2008 update," Hepatology International, vol. 2, no. 3, pp. 263-283, 2008.

[11] European Association for the Study of the Liver, "EASL clinical practice guidelines: management of chronic hepatitis B virus infection," Journal of Hepatology, vol. 57, no. 1, pp. 167-185, 2012.

[12] A. S. F. Lok and B. J. McMahon, "Chronichepatitis B: update 2009," Hepatology, vol. 50, no. 3, pp. 661-662, 2009.

[13] S. L. Friedman, "Preface," Clinics in Liver Disease, vol. 12, no. 4, pp. 13-14, 2008.

[14] F. Imbert-Bismut, V. Ratziu, L. Pieroni, F. Charlotte, Y. Benhamou, and T. Poynard, "Biochemical markers of liver fibrosis in patients with hepatitis $C$ virus infection: a prospective study," The Lancet, vol. 357, no. 9262, pp. 1069-1075, 2001.

[15] K. Patel, D. R. Nelson, D. C. Rockey et al., "Correlation of FIBROSpect II with histologic and morphometric evaluation of liver fibrosis in chronic hepatitis C," Clinical Gastroenterology and Hepatology, vol. 6, no. 2, pp. 242-247, 2008. 
[16] F. Ter Borg, R. A. F. M. Chamuleau, G. N. J. Tytgat et al., "A survey of liver pathology in needle biopsies from HBsAg and anti-HBe positive individuals," Journal of Clinical Pathology, vol. 53, no. 7, pp. 541-548, 2000.

[17] A. Regev, M. Berho, L. J. Jeffers et al., "Sampling error and intraobserver variation in liver biopsy in patients with chronic HCV infection," American Journal of Gastroenterology, vol. 97, no. 10, pp. 2614-2618, 2002.

[18] S. Alam, N. Ahmad, G. Mustafa, A. Shrestha, A. K. Alam, and M. Khan, "Evaluation of normal or minimally elevated alanine transaminase, age and DNA level in predicting liver histological changes in chronic hepatitis B," Liver International, vol. 31, no. 6, pp. 826-832, 2011.

[19] L.-N. Ma, Z.-M. He, Z.-H. Cao, Y.-H. Zhang, and X.-Y. Chen, "Relationship between liver pathology and clinical characters in HBV carriers," Zhonghua Gan Zang Bing Za Zhi, vol. 18, no. 6, pp. 433-436, 2010.

[20] C. M. N. Croagh, S. J. Bell, J. Slavin et al., "Increasing hepatitis $\mathrm{B}$ viral load is associated with risk of significant liver fibrosis in HBeAg-negative but not HBeAg-positive chronic hepatitis B," Liver International, vol. 30, no. 8, pp. 1115-1122, 2010.

[21] Y. Wang, M. Y. Xu, R. D. Zheng et al., "Prediction of significant fibrosis and cirrhosis in hepatitis B e-antigen negative patients with chronic hepatitis B using routine parameters," Hepatology Research, vol. 43, no. 5, pp. 441-451, 2013.

[22] X. D. Liu, J. L. Wu, J. Liang, T. Zhang, and Q. S. Sheng, "Globulin-platelet model predicts minimal fibrosis and cirrhosis in chronic hepatitis B virus infected patients," World Journal of Gastroenterology, vol. 18, no. 22, pp. 2784-2792, 2012.

[23] J.-B. Nousbaum, J.-F. Cadranel, G. Bonnemaison et al., "Clinical practice guidelines on the use of liver biopsy," Gastroenterologie Clinique et Biologique, vol. 26, no. 10, pp. 848-878, 2002.

[24] P. Bedossa and T. Poynard, "An algorithm for the grading of activity in chronic hepatitis C," Hepatology, vol. 24, no. 2, pp. 289-293, 1996.

[25] D. Prati, E. Taioli, A. Zanella et al., "Updated definitions of healthy ranges for serum alanine aminotransferase levels," Annals of Internal Medicine, vol. 137, no. 1, pp. 1-9, 2002.

[26] M. Kumar, S. K. Sarin, S. Hissar et al., "Virologic and histologic features of chronic hepatitis B virus-infected asymptomatic patients with persistently normal ALT,' Gastroenterology, vol. 134, no. 5, pp. 1376-1384, 2008.

[27] M. Mohamadnejad, G. Montazeri, A. Fazlollahi et al., "Noninvasive markers of liver fibrosis and inflammation in chronic hepatitis B-virus related liver disease," American Journal of Gastroenterology, vol. 101, no. 11, pp. 2537-2545, 2006.

[28] A. Y. Hui, H. L.-Y. Chan, V. W.-S. Wong et al., "Identification of chronic hepatitis B patients without significant liver fibrosis by a simple noninvasive predictive model," American Journal of Gastroenterology, vol. 100, no. 3, pp. 616-623, 2005.

[29] W.-K. Seto, C.-L. Lai, P. P. C. Ip et al., "A large population histology study showing the lack of association between ALT elevation and significant fibrosis in chronic hepatitis B," PLoS ONE, vol. 7, no. 2, Article ID e32622, 2012.

[30] W. K. Seto, D. K. Wong, J. Fung et al., "High hepatitis B surface antigen levels predict insignificant fibrosis in hepatitis $\mathrm{B}$ e antigen positive chronic hepatitis B," PLoS ONE, vol. 7, no. 8, Article ID e43087, 2012.

[31] Y. Liu, J. Wang, Y. Huang et al., "Molecular epidemiological study of hepatitis B virus in blood donors from five Chinese blood centers," Archives of Virology, vol. 157, no. 9, pp. 1699-1707, 2012.
[32] C.-T. Wai, J. K. Greenson, R. J. Fontana et al., "A simple noninvasive index can predict both significant fibrosis and cirrhosis in patients with chronic hepatitis C," Hepatology, vol. 38, no. 2, pp. 518-526, 2003.

[33] J. Y. Park, Y. N. Park, D. Y. Kim et al., "High prevalence of significant histology in asymptomatic chronic hepatitis B patients with genotype C and high serum HBV DNA levels," Journal of Viral Hepatitis, vol. 15, no. 8, pp. 615-621, 2008.

[34] J. Fung, C.-L. Lai, D. But, D. Wong, T.-K. Cheung, and M.-F. Yuen, "Prevalence of fibrosis and cirrhosis in chronic hepatitis B: implications for treatment and management," American Journal of Gastroenterology, vol. 103, no. 6, pp. 1421-1426, 2008.

[35] W.-K. Seto, C.-F. Lee, C.-L. Lai et al., "A new model using routinely available clinical parameters to predict significant liver fibrosis in chronic hepatitis B," PLoS ONE, vol. 6, no. 8, Article ID e23077, 2011.

[36] L. E. Adinolfi, M. G. Giordano, A. Andreana et al., "Hepatic fibrosis plays a central role in the pathogenesis of thrombocytopenia in patients with chronic viral hepatitis," British Journal of Haematology, vol. 113, no. 3, pp. 590-595, 2001.

[37] M.-F. Yuen, Y. Tanaka, I. O.-L. Ng et al., "Hepatic necroinflammation and fibrosis in patients with genotypes $\mathrm{Ba}$ and $\mathrm{C}$, corepromoter and precore mutations," Journal of Viral Hepatitis, vol. 12, no. 5, pp. 513-518, 2005. 


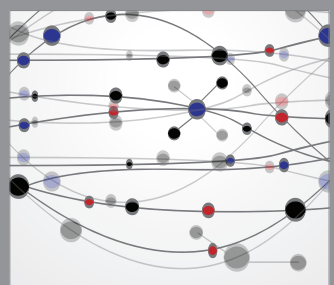

The Scientific World Journal
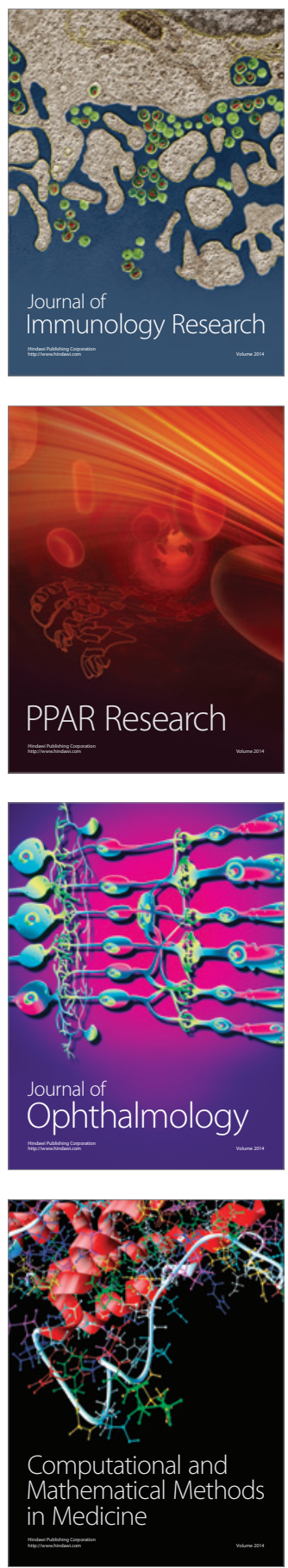

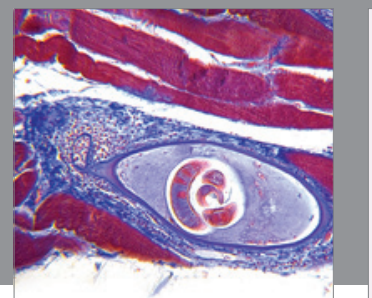

Gastroenterology

Research and Practice
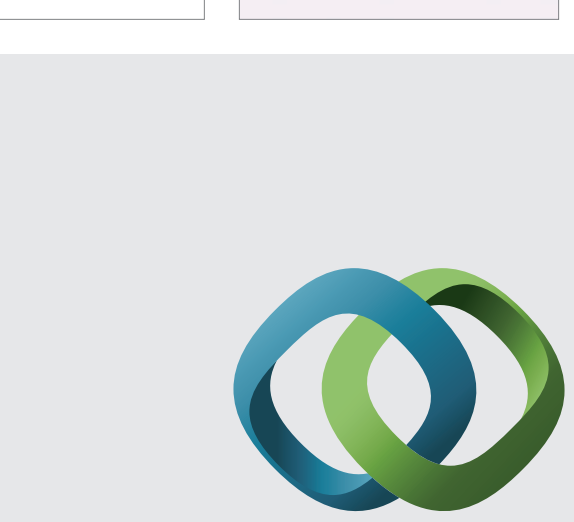

\section{Hindawi}

Submit your manuscripts at

http://www.hindawi.com
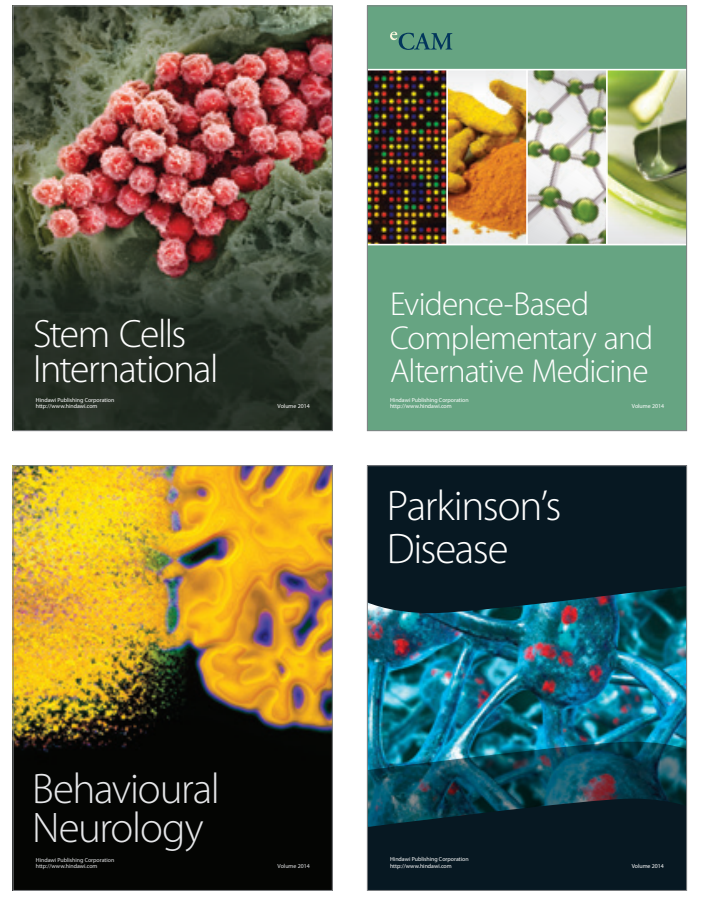
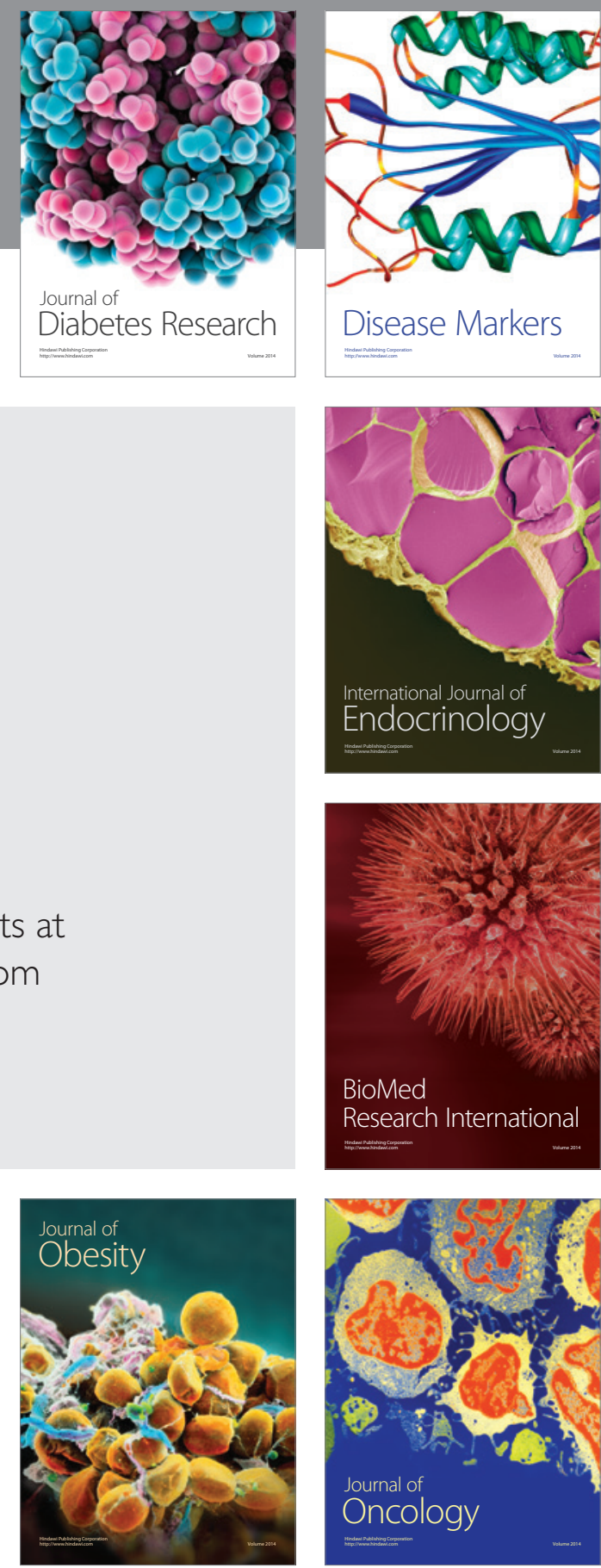

Disease Markers
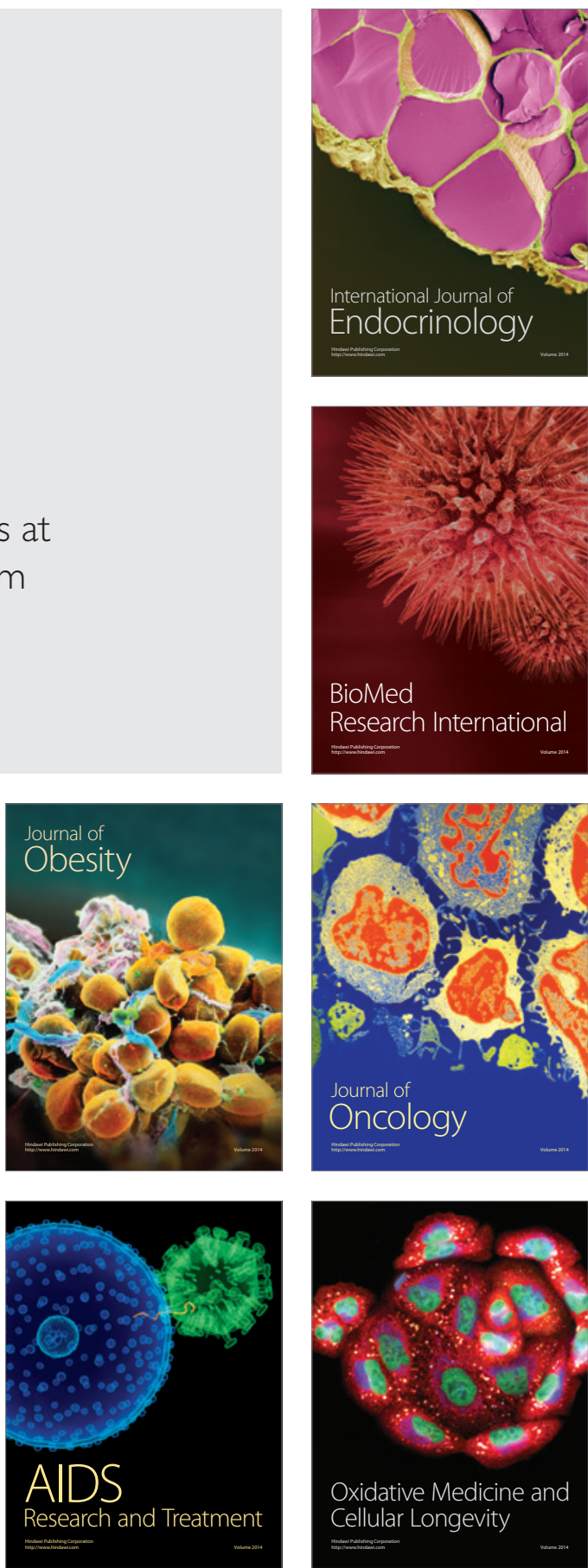